\title{
Advocacia em saúde na enfermagem oncológica: revisão integrativa da literatura
}

\author{
Health advocacy in oncology nursing: an integrative literature review \\ Defensa de la salud en enfermería oncológica: revisión integradora de literatura
}

Franciele Budziareck das Neves ${ }^{1}$ (1) Mara Ambrosina de Oliveira $\operatorname{Vargas}^{1}$ (1) Francielly Zilli ${ }^{1}$ (D)

Daiane Trentin ${ }^{1}$ (B)

Andréa Huhn² (1)

Laura Cavalcanti de Farias Brehmer ${ }^{1}$ (D)

1. Universidade Federal de Santa Catarina,

Florianópolis, Programa de Pós-graduação em Enfermagem. Florianópolis, SC, Brasil.

2. Instituto Federal de Educação Ciências e Tecnologia de Santa Catarina, Departamento Acadêmico de Saúde e Serviço. Florianópolis, SC, Brasil.

Autor correspondente:

Francielly Zilli

E-mail: franciellyzilli.to@gmail.com.

Recebido em 04/04/2020.

Aprovado em 03/06/2020.

DOl:https://doi.org/10.1590/2177-9465-EAN-2020-0106

\section{RESUMO}

Objetivo: Identificar a produção científica acerca do tema advocacia do paciente por enfermeiros no contexto da oncologia. Método: revisão integrativa com busca e seleção dos estudos primários realizadas em fevereiro de 2020 em quatro bases de dados relevantes na área da saúde: Lilacs, SciELO, Cochrane e PubMed. A amostra foi composta por 11 estudos de um universo de 395 artigos. Os 11 estudos foram agrupados em duas categorias. Resultados: A partir da síntese das evidências foi possível elaborar as categorias conforme as temáticas mais abordadas, são elas: "Situações vivenciadas pelos pacientes e enfermeiros em que é necessária a advocacy" "e "Estratégias que aprimoram o exercício de advocacy" que foi subdividida em três subcategorias: Self-advocacy; Sistemas de apoio e advocacy para os pacientes; Comunicação centrada no paciente (CCP) e Tomada de decisão compartilhada (TDC). Conclusões e implicações para a prática: A importância do exercício de advocacy pelos enfermeiros em oncologia é evidente nos estudos, pautando-se na necessidade da comunicação, educação, apoio e reconhecimento da necessidade para intervir quando necessário, considerando a ética como guia para auxiliar na tomada de decisões, reforçando a necessidade de reflexões sobre a temática fortalecendo e embasando tecnicamente a prática profissional

Palavras-chave: Enfermagem; Oncologia; Defesa do Paciente; Advocacia em Saúde; Autonomia Relacional.

\section{ABSTRACT}

Objective: To identify the scientific production on the theme of patient advocacy by nurses in the context of oncology. Method: An integrative review with search and selection of primary studies conducted in February 2020 in four relevant databases in the health area: Lilacs, SciELO, Cochrane, and PubMed. The sample consisted of 11 studies from a universe of 395 articles. The 11 studies were grouped into two categories. Results: From the synthesis of the evidence, it was possible to elaborate the categories according to the most addressed themes: "Situations experienced by patients and nurses in which advocacy is necessary" and "Strategies that improve the exercise of advocacy", which was subdivided into three subcategories: Self-advocacy; Support and advocacy systems for patients; Patient-Centered Communication (PCC) and Shared Decision-Making (SDM). Conclusions and implications for practice: The importance of the exercise of advocacy by nurses in oncology is evident in the studies, based on the need for communication, education, support, and recognition of the need to intervene when necessary, considering ethics as a guide to assist in decision-making, reinforcing the need for reflections on the theme by strengthening and technically basing the professional practice.

Keywords: Nursing; Medical Oncology; Patient Advocacy; Health Advocacy; Relational Autonomy.

\section{RESUMEN}

Objetivo: Identificar la producción científica sobre el tema de la abogacía del paciente por parte de los enfermeros en el contexto de la oncología. Metodo: revisión integradora con búsqueda y selección de estudios primarios realizados en febrero de 2020 en cuatro bases de datos relevantes en el campo de la salud: Lilacs, SciELO, Cochrane y PubMed. La muestra estuvo compuesta por 11 estudios de un universo de 395 artículos. Los 11 estudios se agruparon en dos categorías. Resultados: A partir de la síntesis de la evidencia, fue posible elaborar las categorías de acuerdo con los temas más abordados, que son: "Situaciones que atraviesan pacientes y enfermeros en las que es necesaria la abogacía del paciente" y "Estrategias que mejoran el ejercicio de la defensa o abogacía del paciente" que se subdividió en tres subcategorías: Autodefensa; Sistemas de apoyo y defensa de los pacientes; Comunicación centrada en el paciente (CCP) y Toma de decisiones compartidas (TDC). Conclusión e implicaciones para la práctica: La importancia del ejercicio de la defensa de los pacientes por parte de los enfermeros en oncología se evidencia en los estudios, sobre la base del fortalecimiento de la comunicación, educación, apoyo y reconocimiento de la necesidad de intervenir cuando se menester, considerando la ética como guía para ayudar en la toma de decisiones, reforzar las reflexiones sobre el tema y afianzar los cimientos y la base técnica de la práctica profesional.

Palabras clave: Enfermería; Oncología Médica; Defesa del Paciente; Defesa de la Salud; Autonomía Relacional. 


\section{INTRODUÇÃO}

A definição de advocacia (advocacy) é variável, dependendo do contexto em que é usada. As definições tradicionais de advocacia surgiram a partir da profissão legal em que os direitos de uma pessoa são defendidos, assim como a sua causa. A necessidade de advogar para um paciente está intimamente relacionada com o nível de autonomia que este possa ter. ${ }^{1}$

A advocacia é amplamente definida como um processo de apoio, defesa ou argumentação de uma causa, ideia ou política; por exemplo, os usuários que necessitam de atendimento, tratamento e outros serviços relacionados à condição de saúde podem mobilizar-se politicamente ou usufruir da mídia, solicitando melhorias na assistência médica (cobertura de custos para medicamentos e novas terapias), aumento de investimentos e melhoria na comunicação entre profissionais e pacientes. ${ }^{2,3}$

Pode-se afirmar que a advocacia em saúde é um conceito fundamentado na ideia de defesa contra as iniquidades em saúde. O termo está relacionado com a construção de ações e políticas que visam reduzir tais iniquidades, caracterizadas por condições de vida relacionadas à distribuição desigual de poder, renda e recursos entre países, grupos sociais e pessoas. ${ }^{4,5}$

Na enfermagem, uma série de definições podem ser usadas para descrever a advocacia em saúde, que vão desde agir ou interceder conforme o interesse do paciente, proteção dos direitos do paciente, e uma proteção e conforto para os pacientes incapazes de se comunicar. As múltiplas interpretações da advocacia em saúde podem tornar mais difícil a análise do papel do enfermeiro como um defensor. ${ }^{1}$ Em um estudo ${ }^{6}$ sobre o papel do enfermeiro durante as decisões de tratamento do câncer, foram descritos seis papéis de enfermagem, que incluem: informante da equipe multidisciplinar, educador do paciente, advogado do paciente, gestão de efeitos colaterais, provedor de apoio psicológico e avaliador dos resultados.

Delimitando-se o conceito de advocacy para um contexto específico que relaciona saúde pública e oncologia, percebe-se que o aumento do custo dos cuidados com a saúde, do consumo de produtos de saúde e o aumento da ênfase nos cuidados centrados no paciente, provocaram uma mudança no diálogo relacionado à tomada de decisão sobre o tratamento do câncer entre pacientes médicos e enfermeiros. ${ }^{6}$

No entanto, existem barreiras estruturais relacionadas à defesa da saúde pública que estão relacionadas ao caráter biomédico do modelo hegemônico de atenção à saúde; à pouca discussão que utilize os conceitos de determinação social e desigualdades sociais em saúde para a defesa do direito à saúde; ao pouco envolvimento dos profissionais na defesa da saúde pública; à pouca adesão dos profissionais da saúde à política; ao baixo envolvimento intersetorial e à falta de independência de alguns setores da saúde para a advocacia da saúde pública. ${ }^{4}$

É importante considerar ainda o aumento dos gastos públicos como consequência dos tratamentos mais caros e prolongados, assim como os custos previdenciários decorrentes do afastamento do trabalho, somado às mortes prematuras, anos de incapacidade que reduzem a contribuição social do indivíduo para o desenvolvimento da nação e da sua família. ${ }^{7}$

O câncer é a segunda principal causa de morte em todo o mundo e é responsável por cerca de 9,6 milhões de mortes em 2018. Globalmente, cerca de uma em cada seis mortes é devido ao câncer, e até 2030 pode ultrapassar as doenças cardiovasculares. O custo econômico anual total do câncer em 2010 foi estimado em aproximadamente US\$ 1,16 trilhão. ${ }^{8}$ Portanto, o impacto econômico do câncer é significativo e está aumentando e na medida que as estimativas da doença crescem, também, aumenta proporcionalmente a necessidade do exercício da advocacia na saúde pública, pois é imprescindível para gerir os impactos que a doença pode causar e auxiliar na prevenção de novos casos.

Considerando o exposto, tem-se como as questões de pesquisa: Qual a produção científica acerca do tema advocacia do paciente por enfermeiros no contexto da oncologia, entre os anos de 2010 a 2020? Logo, o objetivo deste estudo foi identificar o que a comunidade científica mundial está discutindo referente à temática da advocacia do paciente em enfermagem, no contexto da oncologia nos últimos 10 anos.

\section{METODOLOGIA}

Trata-se de uma revisão integrativa da literatura (RIL), que visa a síntese de conhecimento, efetuada em seis etapas. ${ }^{9}$ Na primeira etapa, elaboração da pergunta da revisão, foi realizado o aprofundamento teórico a respeito da temática advocacia do paciente na enfermagem oncológica, o que permitiu definir de forma concreta as variáveis de maior significado nesta abordagem de acordo com a literatura existente. Para elaboração da pergunta norteadora da revisão, utilizou-se a estratégia PICO, dessa forma considerou-se $\mathrm{P}$ (paciente) pacientes com diagnóstico de câncer ou sobreviventes do câncer I (intervenção) Advocacy C (controle) não se aplica O (resultado) o exercício do advocacy pelos enfermeiros. ${ }^{10}$

Na segunda etapa da busca e seleção dos estudos primários, escolheram-se as bases de dados de acordo com a pergunta de pesquisa. A pesquisa foi realizada em fevereiro de 2020 , desta forma, considerou-se a possibilidade de inclusão de artigos até a presente data. Utilizou-se as bases de dados eletrônicas do Centro Latino Americano e do Caribe de Informação em Ciências da Saúde (BVS/BIREME)/Lilacs, Scientific Electronic Library Online (SciELO), Cochrane e National Library of Medicine National Institutesof Health (PubMed). A terceira etapa diz respeito à seleção dos termos de busca com base nas palavras que compõe a pergunta de revisão, identificando se o termo apresentava-se como descritor ou palavra-chave. Os descritores utilizados para a coleta de dados seguiram a classificação dos Descritores em Ciências da Saúde (DECS), da biblioteca virtual em saúde e na Medical Subject Headings (MeSH) e foram associados aos termos boleanos OR para distingui-los e AND para associá-los e são os seguintes: Nursing (AND) Medical Oncology (AND) Patient Advocacy, em inglês. Enfermería (AND) Oncologia Médica (AND) Abogacía del paciente, em espanhol. 
Enfermagem (AND) Oncologia (AND) Defesa do paciente, em português. Na identificação dos descritores controlados nas bases de dados selecionadas para a condução da revisão, também foram encontrados sinônimos pertinentes ao termo de interesse como: oncology nursing OR oncology nursing/legislation and jurisprudence; patient advocacy OR advocacy OR health advocacy, também em português e espanhol. Para conectar os descritores e palavras-chaves, além dos operadores booleanos, também foram utilizadas outras técnicas que incluem o uso de parênteses, para estabelecer a ordem no processo de busca e separar os conjuntos de termos e as aspas e colchetes, usadas nos casos de termo composto, formando, desta forma, uma chave de busca conforme o Quadro 1.

$\mathrm{Na}$ quarta etapa que diz respeito à avaliação crítica dos estudos primários incluídos na revisão, o primeiro tratamento foi a aplicação da chave de busca nas bases de dados. Inicialmente, na base SciELO foram identificadas 35 publicações, no Lilacs 177, na Cochrane 10 e no Pumed 173 publicações. O segundo tratamento foi a aplicação dos limites e filtros que contemplaram o intervalo de 10 anos (2010-2020) e pesquisa envolvendo seres humanos, resultando 33 na base SciELO, 47 na Lilacs, 6 na Cochrane e 50 no Pubmed. Justifica-se o recorte temporal de 10 anos visto que foram poucos os estudos publicados nos últimos cinco anos, tornando inviável o corpo de análise desta revisão integrativa da literatura.

O terceiro tratamento foi aplicar os critérios de inclusão e exclusão. Foram incluídos estudos oriundos de pesquisa original, disponíveis na íntegra online; publicados em português ou inglês ou espanhol no recorte temporal citado. Os critérios de exclusão foram artigos publicados em outros meios de comunicação que não periódicos científicos; estudos que não estão disponibilizados on-line para análise, editoriais, artigos de revisão e estudos duplicados.

$\mathrm{Na}$ quinta etapa que se trata da síntese dos resultados da revisão, estes foram agrupados em quadro expositivo, os dados foram digitados e analisados em tabelas no Microsoft Word, utilizando-se de estatística descritiva (Quadro 2) e organizados de acordo com as seguintes variáveis: nome do periódico, título, nome dos autores, ano de publicação, objetivos, país e metodologia.

A etapa de comparação dos dados compreendeu a análise, buscando identificar temas e especificidades nos estudos selecionados, exigindo esforço interpretativo para agrupar os dados e sintetizá-los. Os dados foram agrupados conforme semelhança de temática, facilitando, assim, a apresentação dos resultados em categorias. Por fim, a sexta etapa que constitui a apresentação dos dados constituiu-se na elaboração das conclusões do estudo, assim como as reflexões emergidas no transcorrer da análise. A forma descritiva foi adotada para a análise dos resultados evidenciados, na qual apresentouse a síntese de cada estudo incluído na revisão, assim como comparações entre as pesquisas quando pertinente. $O$ presente estudo não necessitou ser submetido a um Comitê de Ética em Pesquisa por tratar-se de uma RIL e ter utilizado fontes de domínio público para sua realização. Foram seguidos rigorosamente os cuidados éticos na busca, análise e discussão e apresentação dos resultados.

\section{RESULTADOS}

Foram identificados, preliminarmente, 395 estudos por meio da busca nas bases de dados selecionadas e, após selecionar os filtros e limites, o número reduziu para 136 estudos. Posteriormente, foram aplicados os critérios de inclusão e exclusão e os estudos foram avaliados quanto aderência à temática e afinidade à questão de pesquisa. Dessa forma, foram excluídos 47 estudos pois estes eram duplicados, 11 encontravam-se sem resumo $e$ não estavam disponíveis, e por fim, 67 artigos não contemplavam a temática e também foram excluídos. Obteve-se um total de 11 estudos primários para análise.

Quadro 1. Estratégia de busca por base de dados.

\begin{tabular}{|c|c|c|c|}
\hline Base de dados & Estratégia de busca & $\begin{array}{c}\text { Estudos } \\
\text { Encontrados }\end{array}$ & Limites/Filtros* \\
\hline SCIELO & $\begin{array}{l}\text { ((“nursing”[MeSHTerms]) AND (((“medical oncology”[MeSHTerms]) OR } \\
\text { "oncology nursing”[MeSHTerms]) OR (“oncology nursing/legislation and } \\
\text { jurisprudence”[MeSHTerms]))) AND (((“patient advocacy”[MeSHTerms]) } \\
\text { OR advocacy) OR health advocacy) }\end{array}$ & 35 & 33 \\
\hline LILACS & Nursing AND "Medical Oncology" AND "Patient advocacy" & 177 & 47 \\
\hline COCHRANE & Nursing AND "Medical Oncology" AND "Patient advocacy" & 10 & 6 \\
\hline PUBMED & $\begin{array}{l}\text { ((“nursing”[MeSHTerms] ) AND (((“medical oncology"[MeSHTerms]) OR } \\
\text { "oncology nursing”[MeSHTerms]) OR (“oncology nursing/legislation and } \\
\text { jurisprudence"[MeSHTerms]))) AND (((“patient advocacy”[MeSHTerms]) } \\
\text { OR advocacy) OR health advocacy) }\end{array}$ & 173 & 50 \\
\hline
\end{tabular}


Quadro 2. Descrição dos estudos primários

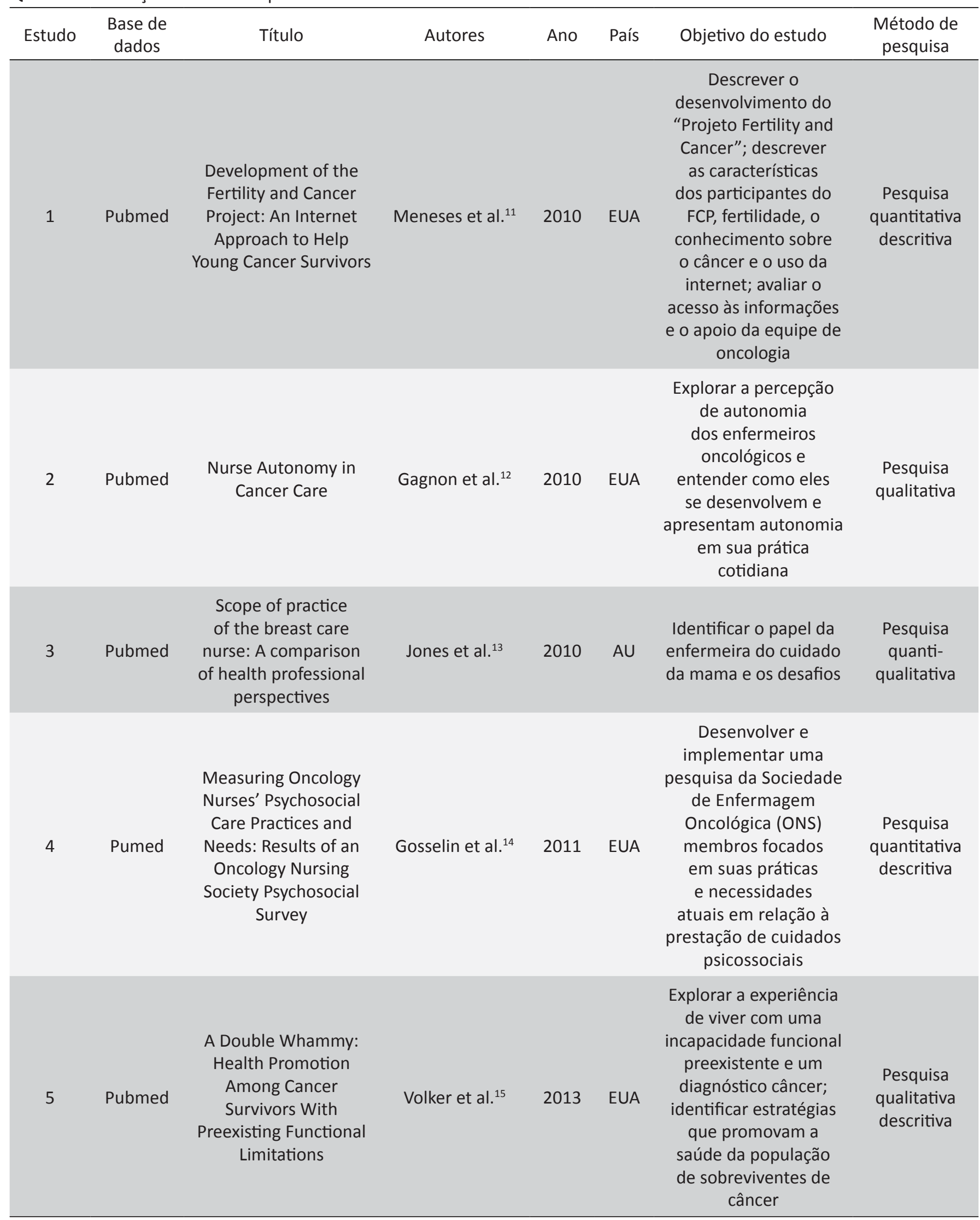


Quadro 2. Continuação...

\begin{tabular}{|c|c|c|c|c|c|c|c|}
\hline Estudo & $\begin{array}{l}\text { Base de } \\
\text { dados }\end{array}$ & Título & Autores & Ano & País & Objetivo do estudo & $\begin{array}{c}\text { Método de } \\
\text { pesquisa }\end{array}$ \\
\hline 6 & Pubmed & $\begin{array}{c}\text { Ovarian Cancer } \\
\text { Survivors' Experiences } \\
\text { of Self-Advocacy: } \\
\text { A Focus Group Study }\end{array}$ & Hagan et al. ${ }^{16}$ & 2013 & EUA & $\begin{array}{l}\text { Explorar as experiências } \\
\text { dos sobreviventes do } \\
\text { câncer de ovário sobre } \\
\text { o "Self-Advocacy" no } \\
\text { manejo dos sintomas }\end{array}$ & $\begin{array}{c}\text { Pesquisa } \\
\text { qualitativa }\end{array}$ \\
\hline 7 & Pubmed & $\begin{array}{l}\text { A Qualitative Analysis } \\
\text { of "Naturalistic" } \\
\text { Conversations in } \\
\text { a Peer-Led Online } \\
\text { Support Community } \\
\text { for Lung Cancer }\end{array}$ & Lobchuk et al. $^{2}$ & 2015 & EUA & $\begin{array}{c}\text { Descrever o conteúdo } \\
\text { das mensagens } \\
\text { presentes em uma } \\
\text { comunidade de suporte } \\
\text { online para câncer de } \\
\text { pulmão nos Estados } \\
\text { Unidos }\end{array}$ & $\begin{array}{c}\text { Pesquisa } \\
\text { qualitativa }\end{array}$ \\
\hline 8 & Pubmed & $\begin{array}{c}\text { Barriers and Promoters } \\
\text { to Participation } \\
\text { in the Era of } \\
\text { Shared Treatment } \\
\text { Decision-Making }\end{array}$ & McCarter et al. ${ }^{3}$ & 2016 & EUA & $\begin{array}{l}\text { Identificar as barreiras } \\
\text { e promotores para } \\
\text { a participação na } \\
\text { decisão de tratamento } \\
\text { do câncer na era do } \\
\text { processo de tomada de } \\
\text { decisão compartilhada }\end{array}$ & $\begin{array}{c}\text { Pesquisa } \\
\text { qualitativa }\end{array}$ \\
\hline 9 & Pubmed & $\begin{array}{c}\text { Communication During } \\
\text { Palliative Care and End } \\
\text { of Life }\end{array}$ & Montgomery et al. ${ }^{17}$ & 2016 & EUA & $\begin{array}{c}\text { Descrever as } \\
\text { características comuns } \\
\text { das percepções de } \\
\text { comunicação durante } \\
\text { os cuidados paliativos e } \\
\text { fim de vida; descrever as } \\
\text { percepções das barreiras } \\
\text { e os facilitadores para } \\
\text { comunicação eficaz dos } \\
\text { enfermeiros }\end{array}$ & $\begin{array}{c}\text { Pesquisa } \\
\text { qualitativa }\end{array}$ \\
\hline 10 & Pubmed & $\begin{array}{c}\text { Engaging Patient } \\
\text { Advocates and } \\
\text { Other Stakeholders } \\
\text { to Design Measures } \\
\text { of Patient-Centered } \\
\text { Communication in } \\
\text { Cancer Care }\end{array}$ & Treiman et al. ${ }^{18}$ & 2017 & EUA & $\begin{array}{l}\text { Desenvolver e } \\
\text { testar questões de } \\
\text { pesquisa para avaliar } \\
\text { as experiências dos } \\
\text { pacientes com a } \\
\text { "Patient-Centered } \\
\text { Communication" no } \\
\text { tratamento do câncer }\end{array}$ & $\begin{array}{c}\text { Pesquisa } \\
\text { qualitativa } \\
\text { descritiva }\end{array}$ \\
\hline 11 & Pubmed & $\begin{array}{l}\text { TBC update: } \\
\text { attitudes of oncology } \\
\text { nurses concerning } \\
\text { pharmacogenomics }\end{array}$ & Dodson $^{19}$ & 2017 & EUA & $\begin{array}{l}\text { Desenvolver um } \\
\text { módulo educacional } \\
\text { interativo e um teste } \\
\text { piloto do instrumento }\end{array}$ & $\begin{array}{c}\text { Pesquisa } \\
\text { qualitativa }\end{array}$ \\
\hline
\end{tabular}

A Figura 1 retrata o fluxograma que descreve o percurso para a seleção dos artigos, baseada no modelo PRISMA. ${ }^{20}$

Com relação à caracterização dos estudos primários que constituíram o corpo de análise, todos foram encontrados na base de dados Pubmed e na Lilacs (duplicados), no idioma inglês, sendo os países de origem os Estados Unidos $(n=10)$ e Austrália $(n=1)$. Quanto ao ano das publicações, o maior número de estudos publicados foi nos anos $2010(n=3)$ e $2016(n=3)$, seguido do ano $2013(n=2)$ e o restante nos anos $2011(n=1)$, $2015(n=1)$ e $2017(n=1)$.

Considerando as características metodológicas, destaca-se a pesquisa qualitativa com oito estudos $(n=8)$, duas pesquisas quantitativas descritivas $(n=2)$ e um estudo quanti-qualitativo $(n=1)$. Os estudos realizados com enfermeiros totalizaram 




Figura 1. Fluxograma da seleção de artigos para a revisão Fonte: dados da revisão, Florianópolis, SC, 2020.

cinco $(n=5)$, enfermeiros e outros profissionais da saúde $(n=1)$ e com pacientes que já apresentaram ou estão com o diagnóstico de câncer foram cinco $(n=5)$. Em relação ao método, três utilizaram entrevistas semiestruturadas $(n=3)$, destas três, uma utilizou também um questionário e outra a observação não-participante. Destaca-se a utilização do grupo focal $(n=3)$, questionário $(n=2)$, pré e pós teste online $(n=1)$, download de mensagens de uma comunidade online $(n=1)$ e entrevista cognitiva $(n=1)$.

\section{DISCUSSÃO}

Os dados encontrados nos diferentes estudos primários incluídos nesta RIL foram sintetizados em duas categorias, intituladas "Situações vivenciadas pelos pacientes e enfermeiros em que é necessário o advocacy" "Estratégias que aprimoramo exercício do advocacy". A categoria "Estratégias que aprimoram o exercício do advocacy" foi subdividida em três subcategorias: Self-advocacy; Sistemas de apoio e advocacy para os pacientes; e Comunicação centrada no paciente (CCP) e Tomada de decisão compartilhada (TDC).

\section{Situações vivenciadas pelos pacientes e enfermeiros em que é necessário o advocacy}

Esta categoria trata das situações e relatos de pacientes e enfermeiros identificados nos estudos em que seria necessária uma intervenção relacionada à advocacia em saúde.

Estudo $^{17}$ apresenta como resultado situações em que foi necessário o enfermeiro exercer o advocacy ou quando o paciente identificou que precisava de um apoio, no entanto, não obteve. Nas situações relatadas, os enfermeiros acreditavam que eram sua responsabilidade ser um advogado para a criança $e$ a família durante o cuidado paliativo e final de vida. A atuação do enfermeiro estava intimamente ligada com a habilidade de se comunicar e o uso dos seus conhecimentos para fornecer orientação antecipatória, resolver problemas com soluções criativas, reconhecer a importância do planejamento de um cuidado avançado para evitar crises, e respeitar as convicções religiosas dos familiares, mas agindo como defensoras das reais necessidades das crianças.

Os enfermeiros do estudo apontaram que para lidar com a evolução dos cuidados paliativos até o final da vida era necessário competência do saber, expansão da essência do cuidar e comprometimento com o advocacy, pois muitas vezes enfrentavam conflitos éticos como a interferência da religião e quando os pais optavam por limitar o fluxo de informações relacionadas com o estado da doença ao seu filho ou quando as crianças se abstiveram de comunicar preocupações sobre o final da vida para proteger seus pais. ${ }^{17}$

Outro estudo ${ }^{15}$ aborda a importância dos profissionais serem os defensores e de proporcionarem apoio social aos pacientes com diagnóstico de câncer e que apresentavam uma condição incapacitante anterior. Os participantes descreveram seus esforços para ser seus próprios defensores e educar os seus cuidadores sobre suas condições pré-existentes. Alguns descreveram experiências difíceis na hospitalização em que os cuidadores eram indiferentes às suas necessidades de autocuidado; outros descreveram problemas com o acesso às instalações do hospital que tinham barreiras significativas para pessoas com mobilidade e deficiência visual. A maioria experimentou preocupação com a capacidade dos oncologistas para recomendar o tratamento do câncer, levando em consideração as outras doenças subjacentes e limitações.

Um estudo ${ }^{3}$ priorizou análise acerca de colocar-se no lugar do outro, pois muitas vezes, os enfermeiros estão sendo solicitados pelos pacientes, "se fosse eu, qual o tratamento que você escolheria?" Esta pergunta retrata o alto nível de confiança dos pacientes com os enfermeiros e posiciona estes profissionais para se tornarem defensores confiáveis para os pacientes, particularmente quando os pacientes não podem advogar para si.

Uma das condições para o enfermeiro exercer o advocacy é a autonomia, sendo descrita como ter liberdade, usando o conhecimento para tomar decisões. Os enfermeiros especialistas em oncologia, que participaram de um estudo, ${ }^{12}$ sinalizaram que estar atualizados sobre a doença e os tratamentos era um pré-requisito para ajudar os pacientes a tornarem-se mais confiantes e confortáveis na tomada de decisões clínicas. Eles descreveram 
como usaram seu conhecimento especializado do câncer para aconselhar os pacientes em estratégias de enfrentamento, ensinar comportamentos de autocuidado e monitorar as respostas às intervenções de tratamento e de enfermagem. Ainda, este estudo reafirma a relação entre autonomia profissional e a defesa do paciente, ou seja, o quanto as tomadas de decisões de enfermeiras oncológicas estão diretamente ligadas às atitudes de advocacy com paciente oncológico.

O avanço no tratamento do câncer traz algumas questões à tona, como por exemplo, a eficácia, o custo e as iniquidades. Nos Estados Unidos, aproximadamente 25\% dos pacientes gastam a maior parte de suas economias durante o tratamento do câncer. O termo "toxicidade financeira" tem sido utilizado para descrever a preocupação atual na área da oncológica. ${ }^{19}$ Neste caso, é fundamental promover a consciência desta "toxicidade financeira", por meio do advocacy. Dessa forma, o autor também versa sobre a importância das informações antecipadas especificando a situação dos testes genéticos e a comunicação de fácil entendimento para o paciente. ${ }^{19}$

\section{Estratégias que aprimoram o exercício do advocacy}

O termo Self-advocacy foi definido como um processo de aprendizagem das próprias necessidades e prioridades enquanto indivíduo com câncer ou sobrevivente e negociação com equipes de saúde, apoio social e outros sobreviventes para atender a essas necessidades. ${ }^{16}$

Um estudo ${ }^{16}$ fenomenológico identificou as principais dimensões e uma definição preliminar de Self-advocacy, em que os enfermeiros podem reconhecer e apoiar quando os pacientes procuram e recebem cuidados consistentes com suas próprias necessidades e preferências.

Ainda, outro estudo ${ }^{15}$ menciona que o conceito de Self-advocacy apareceu na discussão de todas as perguntas nos grupos focais realizados. Os sobreviventes falaram sobre tentar educar os prestadores de cuidados de câncer sobre suas necessidades específicas e incluiu o Self-advocacy como uma estratégia de promoção da saúde e um importante componente de programas de bem-estar para sobreviventes de câncer com preexistência de limitações funcionais. Enfatizada a importância de ensinar as pessoas a gerir os seus cuidados por meio do Self-advocacy e educação, e para encontrar serviços de saúde acessíveis com profissionais sensíveis às suas necessidades.

Outro estudo ${ }^{15}$ apontou a importância da defesa e de apoio social no contexto dos pacientes com câncer que apresentavam uma condição incapacitante, pois a população carente e as pessoas com deficiência experimentam disparidades de saúde sendo mais propensos do que as pessoas sem deficiência a sofrer atrasos e diminuição na obtenção de cuidados de saúde, e portanto, apresentar taxas mais altas de mortalidade relacionadas ao câncer.

Para tanto, exercer o advocacy para o paciente também é educá-lo para se autodefender, e a comunicação tem um papel fundamental neste processo. A troca de informação refere-se à comunicação para avaliar e compreender a informação que os pacientes precisam, facilitar a partilha mútua, e alcançar um entendimento comum. A tomada de decisões reporta-se à comunicação para entender as preferências dos pacientes para a participação na tomada de decisões, deixá-los cientes quando há uma decisão a ser tomada, e envolve-los para que as decisões sejam baseadas na melhor evidência científica e refletir acerca dos seus valores. Fomentar as relações de cura, implica na comunicação que gera confiança, relacionamento, comprometimento e entendimento mútuo sobre os papéis e responsabilidades, permitindo a autogestão da sua doença (por exemplo, controlar os sintomas e efeitos colaterais). ${ }^{18}$

Um dos exemplos de Self-advocacy é retratado neste estudo por mulheres que sinalizaram ter recebido aconselhamento de gestão do sintoma de profissionais de saúde, organizações de defesa, amigos e da Internet, e assim elas descobriram como filtrar e gerenciar as informações para atender suas necessidades e metas. Elas relataram também ser pró-ativas em orientar a equipe de saúde a reconhecer suas próprias prioridades, crenças e valores, a fim de justificar suas próprias escolhas relativas ao seu tratamento e cuidados. As mulheres muitas vezes utilizavam - Self-advocacy nas decisões para reduzir a sua dose de quimioterapia ou não tomar medicamentos por causa de efeitos colaterais indesejados. Ademais, com a sobrevida estendida, muitas vezes é necessário processar a evolução dos desafios por conta própria, fora do ambiente clínico tradicional. ${ }^{16}$

Por sua vez, os sistemas de apoio e advocacy são comunidades de suporte online e são fóruns monitorados que fazem parte da web ou de uma rede social em que as pessoas se comunicam entre si, compartilhando informações. Estas comunidades são monitoradas por profissionais e seus pares, lideradas por regras explícitas de conduta online. Usuários liderados por pares também podem auxiliar na administração dessas comunidades. Este tipo de apoio pode colaborar para minimizar o impacto do estresse na saúde, reavaliando o que pode ser uma ameaça e encorajando comportamentos positivos de enfrentamento. Os usuários de comunidades de apoio online monitoradas frequentemente discutem emoções, buscam e fornecem informações sobre o câncer (informações médicas, tratamento, respostas terapêuticas), além de compartilhar o diagnóstico diário e oferecer apoio espiritual, mas nenhuma terapia é oferecida. ${ }^{2}$

Um estudo ${ }^{11}$ envolvendo 106 mulheres jovens sobreviventes de câncer de mama descreve que $24 \%$ destas afirma ter recebido apoio emocional suficiente de seus prestadores de cuidados em oncologia e $11 \%$, receberam informações suficientes sobre a preservação da fertilidade. Resultados preliminares do projeto "Fertility and Cancer" de que trata este estudo contribuíram para o corpo de conhecimento de jovens sobreviventes de câncer de mama e suas experiências em busca de informações de saúde sobre a fertilidade. Primeiro as mulheres jovens sobreviventes de câncer de mama usam a Internet para buscar informações sobre fertilidade e apesar de apresentar bom nível de escolaridade e ter renda acima da média, muitas não têm acesso imediato à informação por meio de fontes tradicionais, ou seja, com os 
profissionais. E, três quartos dos participantes conheceram o grupo "Fertility and Cancer" por meio de outros grupos de advocacy online e pesquisas na Web, o que sugere que jovens sobreviventes de câncer de mama frequentemente formam uma rede com outras sobreviventes do câncer. ${ }^{11}$ Nesse estudo foi possível identificar que a estratégia dos grupos de advocacy estava voltada para a divulgação do projeto "Fertility and Cancer", reforçando a compreensão de que a advocacia, neste caso, favorece a autonomia das pacientes através do processo de apoio ao ofertar acolhimento e informações necessárias por meio de mídias online.

Quanto aos grupos de apoio, estudo ${ }^{13}$ traz o "The Breast Care Nurse" (BCN) que é um grupo de enfermeiros que desenvolvem um trabalho visando o cuidado do paciente com câncer de mama. A ideia foi formalmente introduzida nos serviços de saúde da Austrália no final de 1990 para facilitar uma melhor continuidade dos cuidados e apoio psicossocial. O papel do enfermeiro é coordenar o atendimento ao paciente, fazendo ligações para outros profissionais, intervindo e defendendo o paciente quando necessário. Mencionado que os enfermeiros também forneciam informações e apoio psicossocial às famílias dos pacientes.

Em estudo ${ }^{14}$ realizado pela Oncology Nursing Society (ONS) com 401 enfermeiros identificou que $60 \%$ destes utilizavam os grupos de apoio como ferramentas e métodos para ajudar os pacientes e famílias, já que estes grupos oferecem orientações (por exemplo, o self-advocacy) e $88 \%$ consideraram os grupos de apoio acessíveis e disponíveis como um recurso psicossocial. Além disso, 35\% dos participantes consideram o enfermeiro o profissional responsável pelo fornecimento de serviços psicossociais na saúde, superando os $33 \%$ que identificaram o assistente social.

Por fim, a comunicação centrada no paciente (CCP) compreende a comunicação entre os pacientes e profissionais e necessita de três atributos fundamentais: consideração das necessidades dos pacientes, perspectivas e experiências individuais; criação de oportunidades para os pacientes para participar de seu cuidado; e fortalecimento do paciente pelo médico. No contexto dos pacientes com câncer, a CCP ajuda os pacientes de várias maneiras, tais como receber notícias difíceis e depois lidar com o impacto emocional de uma doença potencialmente fatal, compreender a informação médica mais complexa, comunicando-se com vários profissionais de saúde, lidar com a incerteza, a tomada de decisões sobre o que importa para si, e a adoção de comportamentos promotores de saúde. ${ }^{18}$

Falhas na comunicação e o não advocacy podem causar consequências como gestão inadequada do sintoma diminuição da qualidade de vida, erros de medicação e mal-entendidos a respeito das preferências dos pacientes e da família para os cuidados. Além disso, a falta de comunicação sobre o planejamento da assistência pode levar a terapias agressivas e indesejadas para o paciente.

Já a Tomada de Decisão Compartilhada (TDC) é um modelo de prestação de cuidados de saúde que postula quatro princípios fundamentais: pelo menos dois médicos participantes e paciente envolvidos; ambas as partes partilham informação; ambas as partes constroem consenso sobre o tratamento preferencial; $\mathrm{e}$ seja alcançado um acordo sobre a implementação do tratamento. Este modelo é relevante na oncologia porque os enfermeiros foram identificados como os principais profissionais que são fontes de informação, controlam os efeitos colaterais da quimioterapia, avaliam os resultados do tratamento, e advogam em nome dos pacientes oncológicos. Dessa forma, o modelo de TDC incorpora tanto o paciente, médicos e enfermeiros. ${ }^{3}$

Os princípios da TDC exigem dos enfermeiros papéis complexos na tomada de decisões tratamento do câncer, que incluem papel: educador do paciente, informante durante a troca de informações entre os membros da equipe de saúde e o paciente e de advocacia para alcançar uma decisão de tratamento que é acordado entre os pacientes, médicos, enfermeiros e outros membros da equipe de saúde. ${ }^{3}$

As limitações do presente estudo estão relacionadas ao número escasso de estudos primários elegíveis para compor a RIL; o levantamento de dados metodológicos, já que alguns estudos não apresentavam de maneira explícita a descrição da metodologia.

\section{CONSIDERAÇÕES FINAIS}

Considerando que, embora o advocacy seja permeado de subjetividades, ainda assim, é possível elencar elementos chaves que vem a ser a estruturação da defesa dos pacientes/ usuários com câncer. Os indicadores do referencial advocacy nos estudos abordados nesta RIL estão relacionados à autonomia do paciente ao buscar seus direitos, à autonomia do profissional em defender seu paciente e ao direito à informação e a comunicação.

A questão da obrigação ética de informar o paciente deve ser inerente ao cuidado já que é um direito do paciente ser informado sobre a sua condição de saúde e assim promover sua autonomia. Logo, é necessário saber administrar os dilemas e problemas éticos que possam emergir quando as informações geram ansiedade e estresse. No entanto, a expansão do papel do enfermeiro, no cuidado ao paciente com câncer, tem aumentando a autonomia no que diz respeito ao processo de tomada de decisão de tratamento do câncer e pode resultar na satisfação dos pacientes.

Apontou-se como fator importante que os achados discutidos neste estudo são todos internacionais, reforçando não só a escassa produção a respeito do tema, mas a lacuna existente principalmente em nosso país. Ainda, considerou-se que o conteúdo destas produções e a relevância da temática no momento atual torna o desenvolvimento deste estudo imprescindível para a produção de futuros estudos que possam abordar esta temática nacionalmente. Ressalta-se ainda que nenhum dos estudos tinha como objetivo de análise o Advocacy, mas esta temática emergiu dos dados conforme o que foi expresso pelos participantes das pesquisas e nos conceitos dos temas abordados. Ademais, torna-se necessária a produção de estudos que permitam aos profissionais quantificar e qualificar o quanto os pacientes/usuários estão sendo defendidos, isto é, quais os 
resultados desta prática e como os indicadores são abordados ou realizados para que a defesa ocorra efetivamente.

\section{CONTRIBUIÇÕES DOS AUTORES}

Desenho do estudo de revisão. Aquisição, análise de dados e interpretação dos resultados. Redação e revisão crítica do manuscrito. Aprovação da versão final do artigo. Responsabilidade por todos os aspectos do conteúdo e a integridade do artigo publicado. Franciele Budziareck das Neves.

Desenho do estudo de revisão. Interpretação dos resultados. Redação e revisão crítica do manuscrito. Aprovação da versão final do artigo. Responsabilidade por todos os aspectos do conteúdo e a integridade do artigo publicado. Mara Ambrosina de Oliveira Vargas

Análise de dados. Redação e revisão crítica do manuscrito. Aprovação da versão final do artigo. Responsabilidade por todos os aspectos do conteúdo e a integridade do artigo publicado. Francielly Zilli

Interpretação dos resultados. Redação e revisão crítica do manuscrito. Aprovação da versão final do artigo. Responsabilidade por todos os aspectos do conteúdo e a integridade do artigo publicado. Andréa Huhn. Laura Cavalcanti de Farias Brehmer. Daiane Trentin.

\section{EDITOR ASSOCIADO}

Marcelle Miranda da Silva

\section{REFERÊNCIAS}

1. Cole C, Wellard S, Mummery J. Problematising autonomy and advocacy in nursing. Nurs Ethics. 2014 jan;21(5):576-82. http://dx.doi. org/10.1177/0969733013511362. PMid:24399831.

2. Lobchuk M, McClement S, Rigney M, Copeland A, Bayrampour H. A qualitative analysis of "naturalistic" conversations in a peer-led online support community for lung cancer. Cancer Nurs. 2015 set;38(5):21-31 http://dx.doi.org/10.1097/NCC.0000000000000207. PMid:25254411.

3. McCarter SP, Tariman JD, Spawn N, Mehmeti E, Bishop-Royse J, Garcia I et al. Barriers and promoters to participation in the era of shared treatment decision-making. West J Nurs Res. 2016 maio;38(10):1282 1297. http://dx.doi.org/10.1177/0193945916650648. PMid:27194634.

4. Cohen BE, Marshall SG. Does public health advocacy seek to redress health inequities? A scoping review. Health Soc Care Community. 2017 jan;25(2):309-28. http://dx.doi.org/10.1111/hsc.12320. PMid:26749000.

5. Farrer L, Marinetti C, Cavaco YC, Costongs C. Advocacy for health equity: a synthesis review. Milbank Q. 2015 jun;93(2):392-437. http:// dx.doi.org/10.1111/1468-0009.12112. PMid:26044634.

6. Tariman JD, Szubski KL. The evolving role of the nurse during the cancer treatment decision-making process: a literature review. Clin
J Oncol Nurs. 2015 out;19(5):548-56. http://dx.doi.org/10.1188/15 CJON.548-556. PMid:26414574.

7. Paiva CJK, Cesse EAP. Aspects related to delay in diagnosis and treatment of breast cancer in a hospital in pernambuco. Rev Bras Cancerol. 2015 mar;61(1):23-30. http://dx.doi.org/10.32635/2176-9745. RBC.2015v61n1.374.

8. World Health Organization. Dados do Global Health Observatory (GHO): câncer [Internet]. Geneva: WHO;2018 [citado 2019 maio 8]. Disponível em: https://www.who.int/en/news-room/fact-sheets/detail/cancer

9. Mendes KS, Silveira RCCP, Galvão CM. Use of the bibliographic reference manager in the selection of primary studies in integrative reviews. Texto \& Contexto. 2019 fev;28(1):13. http://dx.doi.org/10.1590/1980-265xtce-2017-0204.

10. Santos CMC, Pimenta CAM, Nobre MRC. The PICO strategy for the research question construction and evidence search. Revista Latinoame. de Enfermagem. 2007 jun;15(3):508-11. http://dx.doi.org/10.1590/ S0104-11692007000300023.

11. Meneses K, McNees P, Azuero A, Jukkala A. Development of the fertility and cancer project: an internet approach to help young cancer survivors. Oncol Nurs Forum. 2010 fev;37(2):191-7. http://dx.doi.org/10.1188/10. ONF.191-197. PMid:20189924.

12. Gagnon L, Bakker D, Montgomery P, Palkovits JA. Nurse autonomy in cancer care. Cancer Nurs. 2010 maio;33(3):21-8. http://dx.doi. org/10.1097/NCC.0b013e3181c98985. PMid:20357650.

13. Jones L, Leach L, Chambers S, Occhipinti S. Scope of practice of the breast care nurse: a comparison of health professional perspectives. Eur J Oncol Nurs. 2010 set;14(4):322-7. http://dx.doi.org/10.1016/j. ejon.2010.03.006. PMid:20493768.

14. Gosselin TK, Crane-Okada R, Irwin M, Tringali C, Wenzel J. Measuring oncology nurses' psychosocial care practices and needs: results of an oncology nursing society psychosocial survey. Oncol Nurs Forum. 2011 out;38(6):729-37. http://dx.doi.org/10.1188/11.ONF.729-737. PMid:22037335.

15. Volker DL, Becker H, Kang SJ, Kullberg V. A double whammy: health promotion among cancer survivors with preexisting functional limitations. Oncol Nurs Forum. 2013 dez;40(1):64-71. http://dx.doi.org/10.1188/13. ONF.64-71. PMid:23269771.

16. Hagan TL, Donovan HS. Ovarian cancer survivors' experiences of selfadvocacy: a focus group study. Oncol Nurs Forum. 2013 mar;40(2):140-7. http://dx.doi.org/10.1188/13.ONF.A12-A19. PMid:23454476.

17. Montgomery KE, Sawin KJ, Hendricks-Ferguson V. Communication during palliative care and end of life. Cancer Nurs. $2017 \mathrm{abr} ; 40(2): 47-57$. http://dx.doi.org/10.1097/NCC.0000000000000363. PMid:27044058.

18. Treiman K, McCormack L, Olmsted M, Roach N, Reeve BB, Martens $\mathrm{CE}$ et al. Engaging patient advocates and other stakeholders to design measures of patient-centered communication in cancer care. Patient. 2017 set;10(1):93-103. http://dx.doi.org/10.1007/s40271-016-0188-6. PMid:27658673.

19. Dodson $\mathrm{CH}$. TBC update: attitudes of oncology nurses concerning pharmacogenomics. Per Med. 2017 nov; 14(6):515-20. http://dx.doi. org/10.2217/pme-2017-0034. PMid:29749856.

20. Moher D, Liberati A, Tetzlaff J, Altman DG. Preferred reporting items for systematic reviews and meta-analyses: the PRISMA statement. PLoS Med. 2009 jul;6(7):e1000097. http://dx.doi.org/10.1371/journal. pmed.1000097. PMid:19621072. 